\title{
Hypofractionated stereotactic radiotherapy in combination with whole brain radiotherapy for brain metastases
}

\author{
Cesare Giubilei - Gianluca Ingrosso • \\ Marco D'Andrea · Michaela Benassi • \\ Riccardo Santoni
}

Received: 15 May 2008/Accepted: 8 September 2008/Published online: 19 September 2008

(C) Springer Science+Business Media, LLC. 2008

\begin{abstract}
Background The efficacy and toxicity of hypofractionated stereotactic radiotherapy (HSRT) in combination with whole brain radiotherapy (WBRT), for the treatment of 1-4 brain metastases, using a non invasive fixation of the skull, was investigated. Methods Between 04/2001 and 01/2006 30 patients with 44 brain metastases underwent irradiation. Every patient received WBRT $(10 \times 3 \mathrm{~Gy}) ; 41 / 44$ lesions received HSRT boost with a median dose fraction of $6 \mathrm{~Gy}$, the fractionation schemes were $3 \times 6 \mathrm{~Gy}$ and $4 \times 8 \mathrm{~Gy}$; a median total dose of 18 Gy was delivered to the tumor isocenter. Results The median survival period was 9.15 months, the actuarial 1year overall survival and freedom from new brain metastases were $36.6 \%$ and $87.9 \%$, respectively; at univariate analysis Karnofsky Performance Status (KPS) was statistically significant $(P=0.05)$; the actuarial 1-year local control for the $41 / 44$ lesions was $86.1 \%$. No patient had
\end{abstract}

\author{
C. Giubilei · G. Ingrosso $(\bowtie) \cdot$ M. D’Andrea $\cdot$ M. Benassi · \\ R. Santoni \\ Department of Diagnostic Imaging, Molecular Imaging, \\ Interventional Radiology and Radiotherapy, Tor Vergata \\ University General Hospital, V.le Oxford 81, 00133 Rome, Italy \\ e-mail: gianluca.ingrosso@libero.it \\ URL: www.ptvonline.it/dip_immagini.asp \\ C. Giubilei \\ e-mail: c.giubilei@libero.it \\ M. D'Andrea \\ e-mail: marcodan72@tiscali.it \\ M. Benassi \\ e-mail: michaela.benassi@tiscali.it \\ R. Santoni \\ e-mail: riccardo.santoni@uniroma2.it
}

acute or late complications. Conclusions HSRT as a concomitant boost during WBRT is a safe and well tolerated treatment for selected patients with brain metastases.

Keywords Brain metastases .

Hypofractionated stereotactic radiotherapy .

Whole brain radiotherapy - Prognostic factors .

Local control · Radiobiology

$\begin{array}{ll}\text { Abbreviations } \\ \text { HSRT } & \text { Hypofractionated stereotactic radiotherapy } \\ \text { SRS } & \text { Stereotactic radio-surgery } \\ \text { WBRT } & \text { Whole brain radiotherapy } \\ \text { KPS } & \text { Karnofsky performance status } \\ \text { BED } & \text { Biologic effective dose }\end{array}$

\section{Introduction}

The brain is a common site for metastases; the primary tumor spreads to the brain mainly by hematougenous way. Whole brain radiotherapy (WBRT) has been the standard treatment for the past several decades. Recently, stereotactic radiosurgery (SRS) and hypofractionated stereotactic radiotherapy (HSRT) have become common due to their ability to deliver very high doses to a small volume and to obtain high tumor control rates [1]. It seems that from a biological point of view HSRT might have some advantage in comparison to SRS in terms of acute complications [2] and of tumor control rate for lesions larger than $10 \mathrm{cc}$ (or more than $3 \mathrm{~cm}$ of diameter) [3, 4].

Studies demonstrate that the use of up-front WBRT for newly disgnosed brain metastases decrease the risk of methacronous brain metastases in comparison with SRS alone $[5,6]$. 
We retrospectively analyzed 30 patients who underwent hypofractionated stereotactic radiotherapy with a noninvasive fixation of the skull for treatment of solitary or fewer than four metastatic brain lesions, in combination with WBRT.

\section{Methods and materials}

Between April 2001 and January 200630 consecutive patients with 44 metastatic brain lesions underwent irradiation at the radiation oncology therapy unit of the University of Rome, Tor Vergata. Patient and lesions characteristics are summarized in Tables 1 and 2. All the patients received HSRT in combination to WBRT; the median age at the time of the treatment was 63 years; KPS ranged from 70 to 100 (median 90). In 17 patients the primary tumor was controlled; the most common primary was lung (17/30 pts; 57\%); 21 patients presented a single metastasis; 18 did not show any neurogical sign and symptom, while 6 manifested focal neurological deficit, with seizures in two and signs of intracranial pressure in 4 .

Of the 44 brain metastases 41 received fractionated stereotactic radiotherapy. The median tumor volume was $4.8 \mathrm{cc}$ (mean $6.64 \mathrm{cc}$; range $0.4-24.3 \mathrm{cc}$ ) with a median diameter of $21 \mathrm{~mm}$ (mean $25.4 \mathrm{~mm}$; range 10-36 mm).

Treatment was delivered on an outpatient basis. A relocatable non invasive head-mask-system and a biteblock were used to immobilize the patients; a fiducial system (3DLine ${ }^{\circledR}$ ) was used for stereotactic coordinate definition.

Target volume definition was performed on a post-contrast CT and/or MRI; target and critical structures were outlined on a contiguous $2 \mathrm{~mm}$ separated slices. The gross tumor volume (GTV) consisted of the radiographically evident, contrast-enhancing, gross disease; the clinical target volume (CTV) was considered equivalent to GTV; the PTV (planning-target-volume) was defined as the GTV plus a $3 \mathrm{~mm}$ isotropic margin. Treatment plans were produced on Ergo++ 3DLine ${ }^{\circledR}$ treatment planning system. Patients were treated with $6 \mathrm{MV}$ photons using a linac-based stereotactic system; conformal blocking was achieved with a micro-multi-leaf-collimator (3DLine ${ }^{\circledR}$ MMLC).

Two orthogonal portal images were used in order to check set-up alignment; digitally reconstructed radiographs (DRRs), obtained from the CT localization scans, were used as reference images; a matching software was applied to quantify set-up errors between DRRs and portal images.

Forty-one of 44 lesions were treated with HSRT; the median dose fraction was $600 \mathrm{cGy}$ (mean: $654.5 \mathrm{cGy}$; range 600-800 cGy); a median total dose of 18 Gy was delivered to the tumor isocenter (mean: $22.9 \mathrm{~Gy}$; range 16-32 Gy), the median isodose at the periphery was $95 \%$, the mean isodose
Table 1 Characteristics of patients $(n=30)$

\begin{tabular}{|c|c|c|}
\hline & \multicolumn{2}{|c|}{ Patients } \\
\hline & No. & $\%$ \\
\hline Male/female & $14 / 16$ & $46.6 / 53.4$ \\
\hline \multicolumn{3}{|l|}{$A g e^{a}$} \\
\hline$<65$ & 21 & 70 \\
\hline$\geq 65$ & 9 & 30 \\
\hline \multicolumn{3}{|l|}{$K P S^{b}$} \\
\hline$\leq 80$ & 13 & 43.3 \\
\hline$>80$ & 17 & 56.7 \\
\hline \multicolumn{3}{|l|}{ Primary tumor } \\
\hline Controlled & 17 & 56.7 \\
\hline Not controlled & 13 & 43.3 \\
\hline \multicolumn{3}{|c|}{ Extracranial metastases } \\
\hline Exist & 11 & 36.7 \\
\hline None & 19 & 63.3 \\
\hline \multicolumn{3}{|c|}{ No. of metastases } \\
\hline 1 & 21 & 70 \\
\hline 2 & 6 & 20 \\
\hline 3 & 1 & 3.3 \\
\hline 4 & 2 & 6.4 \\
\hline \multicolumn{3}{|l|}{ Primary site } \\
\hline Lung & 17 & 57 \\
\hline Breast & 4 & 13 \\
\hline Colon & 2 & 6.6 \\
\hline Melanoma & 2 & 6.6 \\
\hline Kidney & 2 & 6.6 \\
\hline Others & 3 & 10 \\
\hline
\end{tabular}

${ }^{a}$ Median age $=63$, range 38-88

b Median value $=90$

Table 2 Characteristics of HSRT treated lesions $(n=41)$

\begin{tabular}{lll}
\hline & Lesions & \\
\cline { 2 - 3 } & No. & \\
\hline Primary site & & 53.6 \\
Lung & 22 & 22 \\
Breast & 9 & 4.8 \\
Melon & 2 & 4.8 \\
Kidney & 2 & 4.8 \\
Others & 2 & 9.7 \\
Target volume & 4 & \\
Median & & \\
Range & $4.8 \mathrm{cc}$ & \\
Dose to the isocenter & $0.4-24.3 \mathrm{cc}$ & \\
Median & & \\
Mean & $18 \mathrm{~Gy}$ & \\
Range & $22.9 \mathrm{~Gy}$ & \\
\hline
\end{tabular}


94.8\% (range $88-100 \%$ ). All patients received concomitant WBRT (30 Gy in 10 sessions).

The fractionation schemes were $3 \times 6 \mathrm{~Gy}$ and $4 \times 8$ Gy based upon target volume. Equivalent single fraction doses can be calculated using the Biologic Effective Dose (BED) definition:

$B E D(n, d ; \alpha / \beta)=n d\left(1+\frac{d}{\alpha / \beta}\right)$

where $n$ is the number of fractions and $d$ is the fraction dose.

Assuming $\alpha / \beta$ to be 10 Gy for the tumor tissue, from the condition $B E D(1, d ; \alpha / \beta)=B E D\left(n_{0}, d_{0} ; \alpha / \beta\right)$ where $n_{0}$ and $d_{0}$ describe the chosen fractionation scheme, we find that $3 \times 6$ Gy is equivalent to a single dose $D=12.7 \mathrm{~Gy}$ and $4 \times 8 \mathrm{~Gy}$ to a single dose $D=19.5 \mathrm{~Gy}$.

The aim of the study was to evaluate overall survival, local and intracranial tumor control, toxicity, neurological and overall systemic status.

Follow-up and statistics

Patients underwent clinical follow-up every 3 months with neurological evaluation (KPS). Follow-up ranged between 3.5 and 54.7 months. Every patient was monitored during the treatment and then periodically by neurologic and radiologic examinations.

Follow-up images (MRI) were obtained at 3 month intervals to asses local tumor control and freedom from new brain metastases. Local control was defined as no increase in tumor volume during follow-up MRI; freedom from new brain metastases was defined as no evidence of new lesions in the brain. The results are reported in terms of overall survival and local and intracranial tumor control; toxicity and neurological and overall systemic status were evaluated too.

The statistical analysis was performed using a commercial statistical software package (SPSS 9.0). The actuarial curves were calculated with the Kaplan-Mayer method, according to the interval of the radiation treatment. Univariate analysis was performed using the log-rank test; the Cox-model was used for multivariate analysis.

\section{Results}

\section{Overall survival}

The median survival time for the overall group of patients after combined treatment was 9.15 months (range: 3.5354.77 months). At the time of analysis $2(6.6 \%)$ patients were alive, $4(13.3 \%)$ died for in-field recurrence, $6(20 \%)$

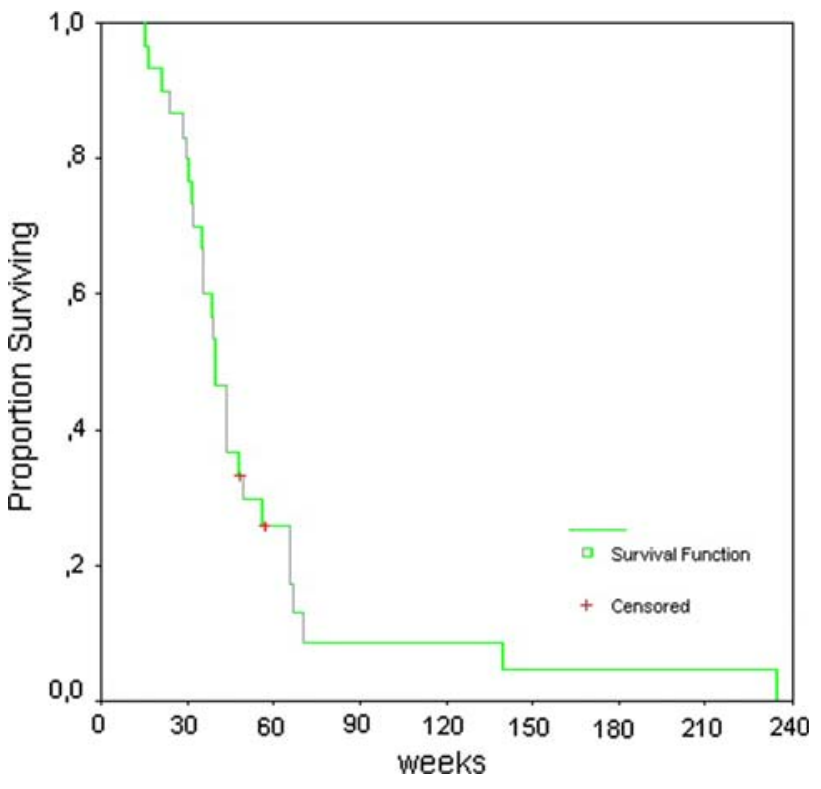

Fig. 1 Overall survival for the 30 patients

for distant intracranial progression and 18 (60\%) for extracranial progression. Actuarial overall survival at 6 months and at 1 year was $86.7 \%$ and $36.6 \%$, respectively (Fig. 1). Univariate analysis considered the following prognostic factors: age ( $<65$ years vs. $\geq 65$ years), KPS ( $\leq 80$ vs. $>80$ ), primary cancer site (lung vs. others) and status (active vs. inactive), presence of extracranial metastases (exist vs. none). The results of this analysis are listed in Table 3 showing that KPS was statistically significant $(P=0.05)$, while the presence of extracranial disease showed only a tendency towards significance $(P=0.07)$ (Fig. 2). KPS remained statistically significant $(P=0.05)$ at the multivariate analysis.

Table 3 Univariate analysis of prognostic factors (30 pts)

\begin{tabular}{lrll}
\hline Characteristics & No. & 1-yr actuarial surv. (\%) & Univariate $P$-value \\
\hline Age & & & 0.42 \\
$<65$ & 21 & 28.5 & \\
$\geq 65$ & 9 & 22.2 & \\
KPS & & & $0.05^{*}$ \\
$\leq 80$ & 13 & 15.3 & \\
$>80$ & 17 & 47 & \\
Primary cancer site & & 0.15 \\
Lung & 17 & 23.5 & \\
Others & 13 & 43.1 & \\
Tumor status & & & \\
Active & 13 & 26.2 & \\
Inactive & 11 & 45.4 & \\
\hline
\end{tabular}

* Statistically significant 


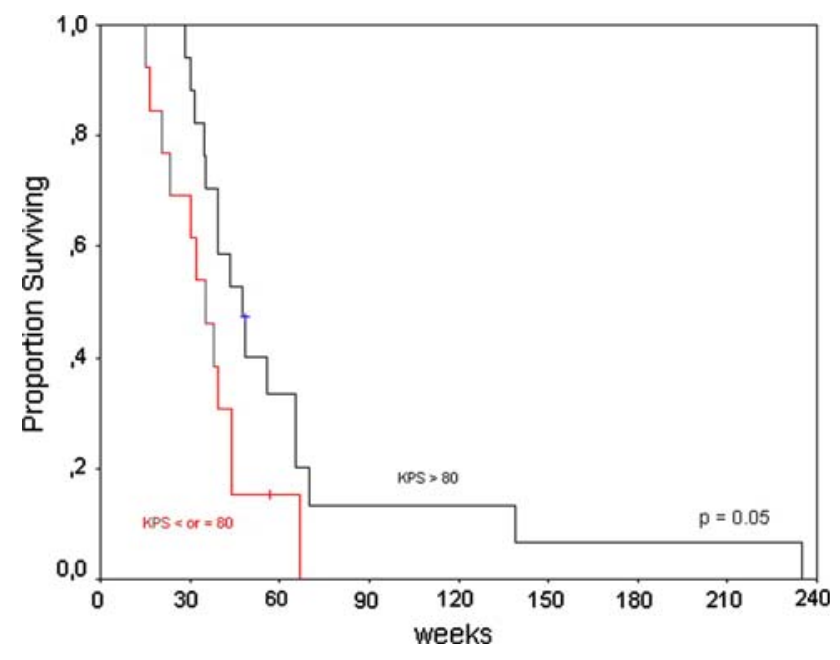

Fig. 2 Kaplan-Mayer curve: overall survival for KPS $\leq 80$ (red line) and KPS $>80$ (black line)

Local tumor control

Local tumor control in the HSRT + WBRT group was achieved in $26 / 30$ patients $(86.7 \%$ ); at the time of analysis 4/41 lesions treated with HSRT had in-field recurrence.

Actuarial local tumor control rates at 6 months and at 1 year were $100 \%$ and $86.1 \%$, respectively.

For the 41 lesions that received WBRT and stereotactic treatment the following prognostic factors were considered: primary cancer site (lung vs. other), tumor size $(\leq 2.1 \mathrm{~cm}$ vs. $>2.1 \mathrm{~cm})$, stereotactic dose $(\leq 18 \mathrm{~Gy}$ vs. $>18 \mathrm{~Gy})$ but none of them was statistically significant (Table 4).

Freedom from new brain metastases

Actuarial rates of freedom from new brain metastases, at 6 months and at 1 year, were $95.1 \%$ and $87.9 \%$, respectively. At the univariate analysis no prognostic factor, for freedom form new brain metastases, was found to be

Table 4 Local control

\begin{tabular}{|c|c|c|c|}
\hline Characteristics & No. & $1-y r$ actuarial surv. (\%) & Univariate $P$-value \\
\hline \multicolumn{3}{|c|}{ Primary cancer site } & 0.25 \\
\hline Lung & 22 & 77.2 & \\
\hline Others & 19 & 92.8 & \\
\hline \multicolumn{3}{|l|}{ Tumor size } & 0.79 \\
\hline$\leq 2.1 \mathrm{~cm}$ & 20 & 88.1 & \\
\hline$>2.1$ & 21 & 80.3 & \\
\hline \multicolumn{3}{|c|}{ Stereotactic dose } & 0.72 \\
\hline$\leq 18$ Gy & 28 & 89.2 & \\
\hline$>18 \mathrm{~Gy}$ & 13 & 83 & \\
\hline
\end{tabular}

Univariate analysis
Table 5 Freedom from new brain metastases

\begin{tabular}{lrll}
\hline Characteristics & No. & 1-yr actuarial surv. (\%) & Unvariate $P$-value \\
\hline Primary cancer & site & & 0.18 \\
Lung & 25 & 91 & \\
Others & 19 & 78.6 & 0.45 \\
No.of brain & metastases & \\
Single & 21 & 83.6 & \\
Multiple & 23 & 90 & 0.49 \\
Primary tumor & & \\
Controlled & 23 & 87.7 & \\
Not controlled & 21 & 88 & 0.8 \\
Extracranial metastases & \\
Exist & 15 & 92.3 & \\
None & 29 & 84.5 & \\
\hline
\end{tabular}

Univariate analysis (44 lesions)

statistically significant. The analysis was conducted for: primary cancer site (lung vs. other), number of brain metastases (single vs. multiple), control of the primary cancer and extracranial control (Table 5).

New brain metastases were observed in seven of the 30 patients, with 13 new lesions (four patients developed one new lesion; one patient four lesions, one patient three lesions, and one patient two lesions); at the time of analysis $6 / 7$ were dead because of the intracranial progression.

Toxicity and neurological status

During the treatment course steroids were administered in order to prevent symptoms. Early symptoms were not observed and no patient suffered because of acute or late complications.

The 18 patients with no symptoms before radiotherapy remaind asymptomatic; of the 12 patients with neurological signs and symptoms $3(10 \%)$ had a complete resolution, four cases $(13 \%)$ a partial one and five patients had no symptomatic benefit.

\section{Discussion}

Several studies have demonstrated that HSRT provides local tumor control and survival benefit for selected patients with brain metastases [7, 8].

HSRT treatment, as delivered in this study, was feasible and well tolerated. All of the patients completed treatment on an outpatient setting and no toxicity was observed during or between treatment sessions. Long-term toxicity and survival were comparable to SRS results; on the contrary a relatively high incidence of acute/early complications after SRS has been reported in the literature [9-11]. 
The actuarial 1-year local tumor control rate $(86.1 \%)$ after HSRT was equivalent to that obtained with SRS series (70-90\%) in several reports [5-12].

As in our patients KPS has been shown to be an important prognostic factor for SRS-treated patients as it is important in the RPA grouping [13, 14].

In the series conducted by Manning et al. 32 patients were treated by HSRT combined with WBRT (30 Gy in 10 fractions); the total dose for HSRT was $27 \mathrm{~Gy}$, delivered in three fractions of $9 \mathrm{~Gy}$. The actuarial survival rate 1 year from HSRT was $44 \%$ and KPS was the main statistically significant factor for survival [15].

The radiobiological rationale favouring the use of fractionation in stereotactic radiotherapy is based on the evidence that the tumor contain a proportion of hypoxic cells which are known to be radioresistant [16]; cell killing is then enhanced by fractionation. Indeed the process of redistribution and reoxygenation within the tumor, taking place between dose fractions, aerates some of the hypoxic surviving cells and they are more likely to be killed by the following one. A second reason supporting a fractionation is based on the fact that radiosensitivity varies during the cell-cycle and the probability of hitting a cell in its most radiosensitive phase is increased if the exposure to ionizing radiation is repeated. Finally it is known from the linear quadratic model of cellular survival that late responding healthy tissues (low $\alpha / \beta$ ratio), are better spared by a fractionated regime than by a single acute dose, for a given level of tumor damage. From the BED formula (1) we can see that the single fraction doses of $12.7 \mathrm{~Gy}$ and $19.5 \mathrm{~Gy}$ give BED values of $66.37 \mathrm{~Gy}$ and $146.53 \mathrm{~Gy}$, respectively for normal brain tissue $(\alpha / \beta=3)$. The corresponding fractionation schemes of $3 \times 6$ Gy and $4 \times 8$ Gy, instead, yield BED values of $54 \mathrm{~Gy}$ and $117.33 \mathrm{~Gy}$, respectively showing a significant decrease in toxicity. However, being high doses more effective at killing radioresistant tumors, the number of fractions has to be kept fairly low. Hypofractionation is a treatment modality for lesions in critical regions, as brainstem, and for large volume targets (>10 cc); there is a strong correlation between the volume of normal brain receiving more than $10 \mathrm{~Gy}$ and side effects of radiosurgery [3, 4]. Hypofractionation has the advantage of giving a large dose-more effective at killing radioresistant tumors-and at the same time has the radiobiological features of fractionation [17].

Fahrig et al. evaluated three different HSRT fractionation schemes for 228 brain metastases: $10 \times 4 \mathrm{~Gy}$, $7 \times 5$ Gy, and $5 \times 6-7$ Gy. Actuarial 6 and 12 months survival was, respectively, $83 \%$ and $66 \%$ with a complete remission rate of $42 \%$. Treatment-related toxicity was influenced by dose fractionation and PTV volume; for metastases $>15 \mathrm{~cm}^{3}$ (diameter $>3 \mathrm{~cm}$ ) $10 \times 4$ Gy is better for acute and late complications [18].
In our series all patients received WBRT in combination with HSRT; we obtained a 1-year freedom form new brain metastases of $87.9 \%$.

A recent study by Sawrie et al. retrospectively analyzed 100 patients treated with SRS for brain metastases in order to define predictors for distant brain failure; indipendent significant factors influencing distant brain failure were the number of lesions $(>3)$, presence or uncontrolled extracranial disease and metastases of melanoma [19].

The randomized study of Aoyama et al. reported results of 132 patients with 1-4 metastases, each $<3 \mathrm{~cm}$, randomized to WBRT plus SRS vs. SRS alone. They found no significant difference in the median overall survival time $(P=0,42)$, while in terms of local and distant intracranial control patients receiving SRS plus WBRT had a better control rate (1-yr actuarial overall control rate $53,2 \%$ vs. 23,6; $P<0,001)$ [20]. Several retrospective studies, despite the inherent biases, have shown that the omission of WBRT doesn't affect overall survival but has a negative impact on local and intracranial control [21-24].

In conclusion we believe that HSRT may be used in the treatment of selected patients with a limited number of brain metastases as a concomitant boost during WBRT; the use of HSRT is based on the classical concepts of radiobiology and does not influence negatively the course of the treatment during whole brain irradiation; it is well tolerated and, in our experience, accepted moreover by informed and collaborating patients.

\section{References}

1. Larson DA, Bova F, Eisert D et al (1994) Current radiosurgery practice: results of an ASTRO survey. Int J Radiat Oncol Biol Phys 28:523-526

2. Hall EJ, Brenner DJ (1993) The radiobiology of radiosurgery: rationale for different treatment regimens for AVMs and malignancies. Int J Radiat Oncol Biol Phys 25:381-385

3. Shiau CY, Sneed KP, Shu HKG et al (1997) Radiosurgery for brain metastases: relationships of dose and pattern of enhancement to local control. Int J Radiat Oncol Biol Phys 37:375-383. doi:10.1016/S0360-3016(96)00497-X

4. Chen JCT, Petrovich Z, O'Day S et al (2000) Stereotactic radiosurgery in the treatment of metastatic disease to the brain. Neurosurgery 47:268-281. doi:10.1097/00006123-20000800000003

5. Sneed PK, Suh JH, Goetsch SJ et al (2002) A multi-institutional review of radiosurgery alone vs. radiosurgery with whole brain radiotherapy as the initial management of brain metastases. Int $\mathbf{J}$ Radiat Oncol Biol Phys 53:519-526. doi:10.1016/S0360-3016 (02)02770-0

6. Pirzkall A, Bebus J, Lohr F et al (1998) Radiosurgery alone or in combination with whole-brain radiotherapy for brain metastases. J Clin Oncol 16:3563-3569

7. Aoyama H, Shirato H, Onimaru R et al (2003) Hypofractionated stereotactic radiotherapy alone without whole-brain irradiation for patients with solitary and oligo brain metastases using 
noninvasive fixation of the skull. Int J Radiat Oncol Biol Phys 56:793-800. doi:10.1016/S0360-3016(03)00014-2

8. Laing RW, Warrington AP, Hines F et al (1993) Fractionated stereotactic extracranial beam radiotherapy in the management of brain metastases. Eur J Cancer 29:1387-1391. doi:10.1016/ 0959-8049(93)90007-3

9. Breneman JC, Warnick RE, Albright RE et al (1996) Stereotactic radiosurgery for the treatment of brain metastases: results of a single institution series. Cancer 79:551-557. doi:10.1002/(SICI) 1097-0142(19970201)79:3<551::AID-CNCR18>3.0.CO;2-2

10. Gelblum DY, Lee H, Bilsky M et al (1998) Radiographic findings and morbidity in patients treated with stereotactic radiosurgery. Int J Radiat Oncol Biol Phys 42:391-395. doi:10.1016/ S0360-3016(98)00230-2

11. Simonova G, Liscak R, Novotny J Jr, Novotny J (2000) Solitary brain metastases with Leksell gamma-knife: prognostic factors for the patient. Radiother Oncol 57:207-213. doi:10.1016/S01678140(00)00267-X

12. Shirato H, Takamura A, Tomita M et al (1997) Stereotactic irradiation without whole-brain irradiation for single brain metastasis. Int J Radiat Oncol Biol Phys 37:385-391. doi: 10.1016/S0360-3016(96)00488-9

13. Cho KH, Hall WA, Gerbi BJ et al (1998) Patient selection criteria for the treatment of brain metastases with stereotactic radiosurgery. J Neurooncol 40:73-86. doi:10.1023/A:1006169109920

14. Auchter RM, Lamond JP, Alexander E et al (1996) A multiinstitutional outcome and prognostic factor analysis of radiosurgery for resectable single brain metastasis. Int $\mathrm{J}$ Radiat Oncol Biol Phys 35:27-35. doi:10.1016/S0360-3016(96)85008-5

15. Manning MA, Robert MC, Benedict HS et al (2000) Hypofractionated stereotactic radiotherapy as an alternative to radiosurgery for the treatment of patients with brain metastases. Int J Radiat Oncol Biol Phys 40:603-608. doi:10.1016/S03603016(00)00475-2

16. Powers WE, Tolmach LJ (1963) Multicomponent X-ray survival curve for mouse lymphosarcoma cells irradiated in vivo. Nature 197:710-711. doi:10.1038/197710b0
17. Brenner DJ, Martel MK, Hall EJ (1991) Fractionated regimens for stereotactic radiotherapy of recurrent tumors in the brain. Int $\mathrm{J}$ Radiat Oncol Biol Phys 21:819-824

18. Fahrig A, Ganslandt O et al (2007) Hypofractionated stereotactic radiotherapy for the brain metastases-result from three different dose concepts. Strahlenther Onkol 183:625-630. doi:10.1007/ s00066-007-1714-1

19. Sawrie SM, Guthrie BL, Spencer SA et al (2008) Predictors of distant brain recurrence for patients with newly diagnosed brain metastases treated with stereotactic radiosurgery alone. Int $\mathbf{J}$ Radiat Oncol Biol Phys 70:181-186. doi:10.1016/j.ijrobp. 2007.05.084

20. Aoyama H, Shirato H, Tago M et al (2006) Stereotactic radiosurgery plus whole brain radiation therapy vs stereotactic radiosurgery alone for treatment of brain metastases: a randomized controlled trial. JAMA 295:2483-2491. doi:10.1001/jama. 295.21.2483

21. Chidel MA, Suh JR, Reddy CA et al (2000) Application of recursive partitioning analysis and evaluation of the use of whole brain radiation among patients treated with stereotactic radiosurgery for newly diagnosed brain metastases. Int J Radiat Oncol Biol Phys 47:993-999. doi:10.1016/S0360-3016(00)00527-7

22. Shehata MK, Young B, Reid B et al (2004) Stereotactic radiosurgery of 468 brain metastases $\leq 2 \mathrm{~cm}$ : implications for SRS dose and whole brain radiation therapy. Int J Radiat Oncol Biol Phys 59:87-93. doi:10.1016/j.ijrobp.2003.10.009

23. Sneed PK, Lamborn KR, Forstner JM et al (1999) Radiosurgery for brain metastases: is whole brain radiotherapy necessary? Int $\mathrm{J}$ Radiat Oncol Biol Phys 43:549-558. doi:10.1016/S0360-3016 (98)00447-7

24. Pirzkall A, Debus J, Lohr F et al (1998) Radiosurgery alone or in combination with whole-brain radiotherapy for brain metastases. J Clin Oncol 16:3563-3569 